12

FINAL REPORT

PROJECT NO. US - 1685 - 89C

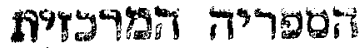

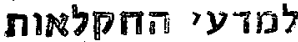

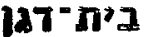

\title{
Optimization of Early Growth in Chickens
}

P.B. Siegel, E.A. Dunnington, Z. Nitsan, I. Nir 
DATE: September 1, 1994

BARD project No. US $-1685-89 \mathrm{C}$

Title:

Optimization of Early Growth in Chickens

Investigators' Names

(Principal listed first)

Siegel, P. B.

Dunnington, E. A.

Nitsan, Z.

Nir, I.

\section{Investigators' Institutions}

Virginia Polytechnic Institute and State University

Virginia Polytechnic Institute and State University

A. R. 0. The Volcani Center

Faculty of Agriculture

The Hebrew University

Project's starting date: August, 1990

Type of Report: 1st Annual

2nd Annual___ Final $X$

fare $B$.

Signature

Principal Investigator signature

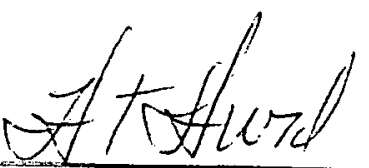

Institution's Authorizing Official

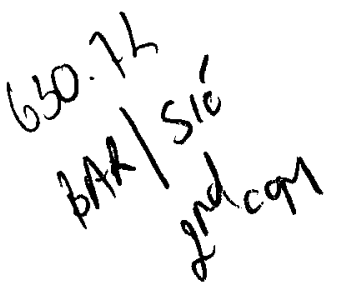

Appendix

6.7 


\section{TABLE OF CONTENTS}

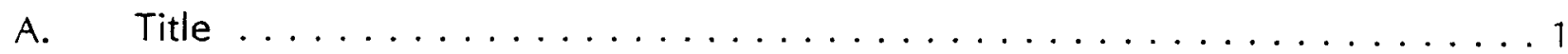

B. Objectives of the Original Proposal $\ldots \ldots \ldots \ldots \ldots \ldots \ldots$

C. Description of Activities and Results $\ldots \ldots \ldots \ldots \ldots \ldots \ldots$ History and Cooperation

Relevant Results and Discussion

Comparative Growth and Development $\ldots \ldots \ldots \ldots$

Factors Prior to Feeding Young Chicks $\ldots \ldots \ldots \ldots$

Social Facilitation and Adaptation . . . . . . . . . . . . 13

Feed Particle Size and Performance ... . . . . . . . . . 17

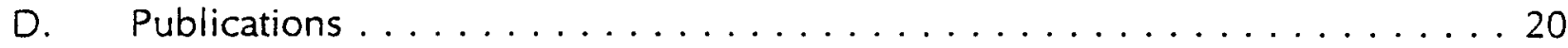

E. Evaluation of Research Achievements . . . . . . . . . . . . . . 22 
Proposal Abstract - Optimization of Early Growth of Chickens

The past 40 years have seen the market age of broiler chickens reduced by approximately one day each year. This trend continues, making the first week an increasing proportion of a broiler's life. During this period, the transition from embryonic absorption of yolk to utilization of exogenous food is accompanied by changes in development of supply (CIT, liver, etc.) and demand (muscles, fat, etc.)

organs. The early period of intense development of supply organs was the main thrust of this research project which was designed to elucidate, via genetic and nongenetic means, relationships among supply organs, demand organs, synthesis of digestive enzymes, intestinal absorption and broiler performance. Methodology included use of known genetic populations and nutritional manipulations. Experiments involving comparative growth and development demonstrated large effects of genetic background on allomorphic relationships, activity of digestive enzymes and blood constituents. These experiments showed that adapting dietary and husbandry practices to specific genetic stocks enhanced their performance. Other experiments showed that limited digestive enzyme synthesis and secretion during the early posthatch period required caution in use of certain exogenous products so as not to activate feedback mechanisms. Feeding of residual yolk to intact chicks and those where the yolk sac had been removed demonstrated genetic differences in response and that lack of endogenous yolk residue was not compensated by exogenous yolk nutrients. Other experiments suggested a "window" during the first week after hatching that was critical to development and functioning that facilitated adaptation to stressors later in life. These experiments were complimented by those designed to evaluate milling practices on GIT growth and function as well as broiler performance. 


\section{BARD FINAL REPORT}

\section{A. TITLE}

\section{Optimization of Early Growth in Chickens}

\section{B. OBJECTIVES OF THE ORIGINAL PROPOSAL}

1. To ascertain genetic and nongenetic factors associated with the digestive system limiting early growth, and develop methods to overcome these limitations in order to enhance broiler performance.

2. To elucidate relationships among early growth, development of supply and demand organs, ontogeny of digestive enzymes and intestinal absorption within and between genetic stocks.

3. To evaluate feasibility of stimulating development of the GIT, appetite, absorption of nutrients and growth rate of the newly hatched chick by dietary manipulation.

\section{DESCRIPTION OF ACTIVITIES AND RESULTS}

Reducing age to achieve market weight is important in the economics of broiler production. Not only is less feed diverted to maintenance when broilers reach market weight at younger ages, but there are additional benefits of more efficient utilization of facilities and equipment. Market weight of broilers has increased in recent years because of shifts from whole-bird to cut-up to value added product. Yet market age continues the trend of the past 40 + years of being reduced by approximately one day per year emphasizing an increasing importance of early life (the first two weeks posthatch) on 
broiler performance.

This project was designed to investigate relationships among early growth, development of supply (e.g., pancreas, !iver, intestine) and demand (e.g., muscle, fat) organs, ontogeny of digestive enzymes, and intestinal absorption of chickens. The first two years of the project were devoted to development and transfer of procedures between laboratories. These efforts, which were documented in annual reports of the project, demonstrated that physiological stages were more important than chronological ages in optimizing early growth of chickens. Also demonstrated was the influence of genetic and nongenetic factors during the early posthatch period on growth and development of the digestive and immune systems.

As planned, the first year of the project provided the basis for research conducted during the second year of the project. The second year provided the basis for the final phase of the project. This orderly approach which was outlined in the project proposal allowed us to capitalize on new items such as the role of social facilitation, milling properties, and late stages of incubation on the genetic-nutrition interface in optimization of early growth. During April, 1992, Siegel visited Israel for a joint review of USA and Israeli experiments. This exchange included designing subsequent experiments to be conducted to complement results from all cooperating units. In September, 1992, the cooperators from the three groups again meet for discussion, the venue being the 19th World's Poultry Congress in Amsterdam. Cooperation was facilitated further during 1993 when Nitsan spent a six-month period with the USA group.

Interactions among the cooperating investigators on this project are evidenced by 
the number of coauthored refereed journal papers listed at the end of this report. Also, involvement of graduate students in the research had multiple and mutual benefits. Students were exposed to scientists from other institutions and a multidisciplinary approach to an area not only of scientific interest, but also with an agricultural and economic impact. In this report we have grouped published and unpublished results into topical areas that address the objectives of the project. When further details are available from published reports, identification is given by listing the number corresponding to it in the publication section of this report.

\section{Comparative Growth and Development}

Growth, one of the most important traits in commercial poultry production, has been widely studied. Selection for body weight modifies the length of autoaccelerated growth phase by changes in the points of inflection and asymptotes. Postnatal body weight is determined by distribution of growth among organs. Comparisons among growth selected populations and crosses among them showed that growth of organs was not proportional to body weight, varied with age, and was amenable to alteration by selection. There is differential growth among supply and demand organs. Because this project was concerned with the early posthatch period, several experiments concentrated on development of the CIT. The GIT has a major role in inducing growth, and during the early posthatching period segments of the GIT increase in size more rapidly than the whole body. Synthesis of digestive enzymes in the pancreas which is limited at hatch increases to a maximum at about 10 days, an age when relative growth rate is near its maximum. The GIT has been implicated as a limiting factor in food intake and growth 
of meat chickens. This is because of the high correlation between body weight and feed intake and because force-feeding trials show that broilers eat at near gut capacity. Therefore, even if feed intake could be increased, low secretion of digestive enzymes during the early period posthatch may limit digestion and hence feed consumption and growth. This conundrum requires further investigation.

Stocks differing in growth rate were used within each experiment presented in this section. Using lines that have undergone selection for high or low juvenile body weight and sublines where selection had been relaxed, data were obtained for body weight, feed consumption, and feed efficiency [3]; organ growth, DNA, RNA, and protein content [4]; and digestive enzymes in the pancreas and intestinal contents [5]. Chicks used in these experiments were fed the low protein [20\%] and energy $2658 \mathrm{kcal} \mathrm{ME} / \mathrm{kg}$ ] diet under which selection had been conducted.

In [3], feed consumption between each selected line and its relaxed subline was equalized by paired feeding. Ad libitum controls were also maintained. At 21 days of age, differences between the selected lines for ad libitum-fed chickens were $404 \%$ for body weight, $279 \%$ for feed intake, and $138 \%$ for feed efficiency. Respective percentages for the high and low relaxed lines under ad libitum feeding were 267, 223, and $121 \%$. When chicks of the line selected for high weight were pair-fed, growth was unimpeded and feed efficiency improved. When chicks of the low-weight relaxed line were pair-fed, feed efficiency also improved, but growth was reduced and appetite development inhibited. Hyperphagic behavior was observed in the line selected for high weight. In the low-weight selected line, the chicks exhibited hypophagia. Although 
residual feed consumption was unchanged by selection for body weight, variation was present for this trait, with lower values under restricted than ad libitum feeding. Thus, it appears that residual feed consumption forms a source of variation in feed efficiency that is not correlated with body weight. The response to feed restriction by the high-weight lines was consistent with that generally observed with commercial broilers where mild feed restriction may improve feed utilization considerably with only a minimal impediment to growth.

In [4], we measured growth of organs relative to body weight and cell protein, DNA, RNA, and cell unit size of breast muscle, liver, and small intestine in the lines and feeding regimes described above. Comparisons at common chronological ages and common body weights revealed that supply organ weights, especially that of the small intestine, were associated with subsequent growth of demand organs. Although the upper GIT was also important in this respect, it was more susceptible to influences such as feed intake. Selection for juvenile body weight resulted in correlated changes in cell size of breast muscle but not liver and small intestine. Muscle increased posthatch as cells underwent hypertrophy but liver and small intestine grew chiefly by hyperplasia. The endodermally derived GIT with its hyperplasic growth was more influenced by environmental factors such as feed allocations while mesodermally derived muscle had hypertrophic growth which was a function of accumulating intercellular proteins.

The same lines and feeding regimes were used in an experiment where amylase, trypsin, chymotrypsin, and lipase in the pancreas and small intestine contents were measured at 8 and 21 days of age [5]. Comparisons between selected lines at common 
ages revealed higher enzyme levels for high- than low-weight lines. When comparisons were made at common body weights there were no differences between lines suggesting that correlated responses in feed intake were mediating the regulation of digestive enzyme levels in the pancreas and in intestinal chyme of growth-selected lines of chickens. They suggest that resource allocations varied primarily according to life cycle demands and biological function rather than chronological age. Chicks from high-weight lines had more elevated enzyme levels after a mild feed restriction than those with ad libitum access to feed. It was hypothesized that hyperphagia associated with the highweight lines in combination with a mild feed restriction and the associated meal feeding stimulated synthesis and secretion of digestive enzymes. Mechanical stimulation of the intestinal wall stimulates synthesis of digestive enzymes. Because of their hypophagic behavior, less stimulation may have occurred in low-weight line chickens with a resulting reduction of synthesis and secretion of digestive enzymes. The unique combination of meal feeding which occurs with either a mild restriction or intermittent feeding in the hyperphagic chick contributed to an increased mechanical stimulation of the GIT and contributed to increased synthesis of digestive enzymes. The absence of hyperphagia in the low-weight lines suggests that meal feeding, and not mild feed restriction per se, may be the elicitor of higher digestive enzyme levels.

Body weights, weights of digestive organs, and activities of digestive enzymes were obtained from hatching to 14 days of age in broiler and in egg-type stocks [6]. Relative daily feed consumption and growth rate were considerably higher in broilers than in egg-type stocks. Relative daily intake peaked at $30 \%$ of body weight on day 3 
posthatch in broilers and at $20 \%$ of body weight at 6 days of age in egg-type stocks. After peak there was a gradual decrease in both stocks. Corresponding values for growth rate were 20 and $14 \%$. Allometric growth of small intestine, liver, and intestinal contents was greater in broilers than egg-type stocks. In contrast, growth of the pancreas was less for broilers. There were differences in the rate of development of pancreatic digestive enzymes between the stocks with total activities relative to body weight suggesting that while they may be limiting in the broilers, they may exceed a need to digest the amount of feed consumed by egg-type stocks. The much higher relative feed consumption of broilers was reflected in their amount of intestinal contents which was not compensated by commensurate increases in pancreas size or digestive enzyme activity. From these results it appears that exogenous supplementation of enzymes would have greater potential in broiler than egg-type chicks.

Nir [unpublished] measured digestive organs, intestinal disaccharidases and some blood metabolites in broiler and egg-type chickens at intervals from hatching to 21 days of age. The objective was to secure information on the activity of the intestinal brushborder disaccharidases responsible for the breakdown of disaccharides [mainly maltose] obtained from starch digestion. In both stocks the activity of maltase and saccharase was very low in the duodenum. Broilers hatched with a reserve of disaccharidases activity exceeding dramatically the activity observed in egg-type chicks. The activity of both enzymes declined with age, much more in broiler than in egg-type chicks. From day 7 to the end of the experiment (day 21), the activity in egg-type chicks exceeded markedly that observed in broilers. The $\mathrm{pH}$ in GIT contents was similar in both stocks. Plasma 
total protein, albumin, and globulin were lower in the broilers than in egg-type chicks. In both lines plasma calcium concentration dropped after hatch and was very low to day 3 after which it stabilized at a higher concentration in egg-type than broiler chicks.

There were no differences between lines in phosphorus concentration which increased from a low of $2 \mathrm{mg} / 100 \mathrm{ml}$ posthatch to a plateau of about $6 \mathrm{mg} / 100 \mathrm{ml}$ on day 10 .

Although on days 1 and 3 posthatch glycemia was higher in broilers, from days 7 to 21 , it was much lower in broiler than in egg-type chicks. This finding corresponds to the lower disaccharidases found in the intestine of the broiler chicks. No differences were found between stocks for triglycerides, cholesterol, uric acid, lactic dehydrogenase, alkaline phosphatase, or $\beta$-hydroxy butyric acid dehydrogenase.

This series of experiments conducted with a range of stocks differing in growth rate demonstrate profound effects of genetic background not only on body weight, but on allomorphic relationships, digestive enzyme activity, and certain blood constituents. The results imply that dietary and husbandry practices adapted to specific genetic stocks have a role during the early posthatch period which is becoming a greater proportion of the life of a broiler, and can also influence subsequent performance.

\section{Factors Prior to Feeding Young Chicks}

The time for a chick embryo to develop and hatch, and its subsequent growth is influenced by many genetic and nongenetic factors. For example, there is both additive and nonadditive genetic variation for time for chicks to pip through the air cell, pip through the shell, and to emerge from the egg. During the period just prior to and shortly after hatching, dramatic changes occur in metabolism of the chick. The 
experiments discussed in this section of this report overlap those covered in the preceding section because in several cases measurements were made for organ growth and digestive enzyme activities.

The vitelline residue, commonly called residual yolk, compliments exogenous nutrient intake during the first few days posthatch and is the nutrient source for the chick until exogenous nutrients are available. Therefore, transition of the digestive system from using embryonic yolk source to requiring exogenous feed is important for maximization of early growth. An experiment [1] conducted early in this project compared internal organs of cockerels from three genetic lines known to differ in growth and in feed consumption patterns. Data were obtained at hatch and through 15 days of age for these traits and for activities of digestive enzymes. On day 15 body weights from the fastest growing line were eight times greater than those from the slowest growing line.

Differences among lines were found for weights at hatching and for growth patterns (both absolute and relative to body weight) of the vitelline residue, heart, lungs, liver, pancreas, crop, proventriculus, gizzard, and segments of the small intestine. Line differences were also evident for levels of trypsin, chymotrypsin, and amylase in the pancreas and contents of the small intestine. Ranking of lines for these traits varied with age. In all lines weights of the small intestine, liver, and pancreas increased relatively more than did total body weight during the first week posthatch, after which the relationship reversed. That the fastest growing line had, relative to body weight, the least residual yolk at hatch suggested considerable complexity for the need and use of this material during the embryonic and transitional periods. Also, segments of the GIT 
relative to body weight were largest in this line, a ranking that did not persist. Similarly, the dynamics among lines changed for digestive enzymes. These results demonstrated that genetic effects could have important implications in both broiler breeding and broiler production and a need for their consideration along with other factors in research in this area.

A body of literature has been developing of the efficiency of lipid transfer from the yolk sac to the embryo. Fertility, hatchability, and their relationships to age of dam, egg components, embryo growth, and embryo lipid transfer were studied in a broiler breeder line segregating at the sex-linked feathering locus [2]. Data were obtained for 8 weeks commencing when pullets were at $50 \%$ lay (28 weeks of age) and again for 5 weeks commencing when pullets were 64 weeks of age. Early season increases in hatchability of eggs were due to a reduction in embryo deaths between days 1 and 7 of incubation. No late seasonal declines in either fertility or hatchability were observed. Although less proficient in young dams, embryo lipid transfer was not directly associated with the lower hatchability of their eggs. Egg weight, weights of 18-day embryos, and embryo:egg ratios increased with parental age. Also with age, there were increases in shell, albumen, and yolk weights. Heavier albumen weight was due to increased moisture content, and those for shell and yolk were due to dry matter accumulation. Changes in yolk:albumen ratios reflected large increases in relative yolk weight, on a wet and dry weight basis, that were associated with age of dam. Eggs from late-feathering dams were heavier than those from early-feathering ones. Heavier embryos by 18 days of incubation were attributed to the transfer of albumen from the egg to the embryo. 
To further explore the role of residual yolk on growth and development, experiments were conducted with chicks whose yolk sacs were removed at hatch and intact chicks [15]. Chicks were then fed ad libitum or intubated with residual yolk or a mixture of vegetable oil and soybean meal. Surgical removal of the yolk sac at hatch reduced early body weight gains. Intubating chicks with a mixture of soybean meal and vegetable oil or with residual yolk obtained from other chicks at hatch, increased body weights and enhanced development of the liver and pancreas. Whereas intubation with residual yolk reduced absorption of yolk sac contents, absorption was similar for chicks fed mash ad libitum or intubated with the soy-vegetable oil diet. After cessation of yolk intubation, absorption of residual yolk resumed. Removal of the yolk sac decreased amylase and lipase activity in the pancreas. In the intestinal chyme, activity of amylase, trypsin, and chymotrypsin was reduced by yolk sac removal. Intubation with yolk increased enzyme activity (amylase excepted) in the pancreas and/or intestinal chyme only in yolk-removed chicks. There was evidence that contents of the yolk sac did not enter the intestine via the yolk stalk, but was absorbed directly via the blood.

From experiments described this far in this report it is evident that digestive enzyme synthesis and secretion is limited during the early posthatch period. Industrial enzyme products may have potential as feed additives and some laboratories have reported beneficial effects for poultry. Amylase and protease from bacterial origin were supplemented to a diet fed broilers from hatch to 14 days [13]. Traits measured included GIT development and digestive enzyme activities, as well as possible carry-over effects to market age. 
Five treatments were studied: 1-diet not supplemented with enzymes; 2supplementation with 250 ppm enzyme preparation I (EI250); 3-supplementation with 1000 ppm enzyme preparation I (El1000); 4- supplementation with 250 ppm enzyme preparation II (Ell250); 5-supplementation of 1000 ppm enzyme preparation II (Ell1000). Enzyme preparations were supplemented to day 14 . The enzyme preparations were produced by Bacillus subtilis and Penicillium emersonii. Activities of the supplemental enzymes were determined as follows: Amylase was determined as in the digestive tract and expressed as glucose produced per min from starch per mg enzyme premix. The values were 0.38 and $0.96 \mathrm{mg}$, for El and Ell respectively. Protease total activity was measured by recording tyrosine produced from casein. The values found were 38 and 48 رeq per $\mathrm{mg}$ for enzymes preparations $\mathrm{El}$ and Ell. Differences in in vitro activities of the supplemented enzymes did not correspond to the effects observed in vivo. Although the in vitro activity of amylase and proteases in Ell preparation was 2.50 and 1.25 higher than that observed in the El preparation, effects of the two preparations observed in vivo did not differ. Supplementation of the enzymes preparation did not affect digestibility, feed intake, growth rate or feed utilization. The lack of effect on performance was not due to the inactivation of the enzymes in the digestive tract because effects of the enzymes supplementation were observed in the GIT i.e., a reduction in the weight of the small intestine accompanied by an increase in the weight of its contents, and by a reduction in the content of the gizzard. The $\mathrm{pH}$ of the intestinal content was reduced by the enzyme supplementation. Supplementation of exogenous amylases and proteases depressed the production of and/or secretion of pancreatic amylase and proteases 
(trypsin, and chymotrypsin). It may be proposed that secretion of pancreatic enzymes is regulated by feedback mechanisms triggered by concentration of their products. If correct, supplementation of diets with enzymes should be made with caution, especially when the activity of the supplemented enzymes resembles that of the endogenous pancreatic ones.

\section{Social Facilitation and Adaptation}

The period immediately after hatching is critical to the life and health of a chick. With current production practices there is no hen-chick bonding - chicks are raised with age contemporaries. Little is known about the effects of stressors during the first week of life and subsequent performance of broiler populations. Also, prompt consumption of food and water by chicks is important in broiler production. Experiments were conducted to measure effects of social facilitation on behavior, body and organ weight, and digestive enzyme activity in stocks known to differ in growth and feed consumption. Other experiments were concerned with the effect of yolk sac removal on food preferences and the influence of mild feed restriction during the critical period of supply organ growth on stress responses.

Ingestive behavior, livability, and body weight were measured in lines of chickens selected for high and low juvenile body weight, reared in cages and floor pens as lineseparate (S) and line-intermingled (I) flocks [7]. There were three trials. Percentages of chicks eating and drinking were similar or greater in I than S flocks. There were no trial by $1-S$ interactions for these behaviors at 3, 14, and 28 days of age. On day 7 , however, these interactions were significant. From experiments described earlier in this report, we 
know that this is the age when there are considerable differences between populations (these lines included) in development of supply organs and digestive enzyme syntheses and secretion. High-line chicks reared in cages were heavier in 1 than S flocks, whereas, no influence on body weight was evident under floor rearing for high-weight or for lowline chicks. Mortality for high-weight line chicks was very low. Anorexia has been reported in the low-weight line and death from starvation was greater in that line for $S$ than I flocks in cages. This difference in mortality was not observed in floor pens. The results of this research suggest that ingestive behavior, livability, and growth may be influenced by intermingling chicks from genetic lines that differ in eating behaviors. The degree of influence, however, is not independent of age, housing environment, and genetic stock.

The effect of 3-day-old 'guides' of broiler or White Leghorn chickens on posthatch behavior (eating, drinking, standing, and sitting), growth rate, and livability were measured in broiler and Leghorn chickens [8]. Four trials were conducted. One trial was in cages and three were on a litter floor. Although the guides did not influence the behaviors of either breed reared in cages, growth of broilers, but not of Leghorns, was depressed by the presence of broiler guides. In floor experiments, the presence of guides of both breeds increased ingestive behavior (eating and drinking), with reduced growth of broilers reared with broiler guides consistent with that observed in cages. Whereas in unstressed chicks' vitality was not affected by the guides, cold stressing of Leghorn chicks in the hatcher markedly increased the positive effect of the guides on ingestive behavior, vitality, and growth rate. Responses of the posthatched chickens to 
3-day-old guides were dependent on housing, management, genetic stock, and stressful settings.

5

The effect of removal of residual yolk at hatching on growth and feeding behavior was measured in the lines of chickens that had been divergently selected for high and low juvenile body weight [14]. From each line, intact chicks and those with yolk sacs removed were assigned to an all-mash feeding regime or given a choice between mash and residual yolk. Regardless of treatment high-weight line chicks were heavier and consumed more feed than those from the low-weight line. On day 5 and thereafter, chicks given a choice were heavier than those eating mash only. Percentage of residual yolk consumed in choice feeding increased during the first 2 to 3 days after hatch, remained constant for the next 2 days and then decreased. Development of the GIT was similar in intact and yolk sac-removed chicks from the low-weight line. In the highweight line, however, intact chicks had the more developed GIT suggesting that they were able to utilize endogenous yolk more efficiently than exogenous yolk. Because chicks from the low line consumed more yolk than those from the high line and the latter consumed more mash then the former, perhaps high-weight line chicks had greater capabilities to digest the carbohydrate part of the diet while low-weight chicks were more efficient in digestion of lipid. Moreover, chicks from the low line presented with a choice reduced their energy intake and maintained protein consumption, as compared to chicks eating only mash. Those chicks also ate less but gained more weight to 4 and 7 days of age than controls, suggesting that chicks from the low line were not as efficient as those from the high line in the digestion of the carbohydrate part of the mash while 
protein may be well digested in both lines. These results may explain why "exogenous" yolk improved growth even in the fast growing line. This experiment suggests that yolk residue has a determining role in the initiation of growth (during the first week after hatch) which cannot be compensated by feeding exogenous yolk nutrients in faster growing individuals.

It is evident from the experiments described thus far that the middle to the end of the first week after hatching is critical in the development and functioning of supply organs. An experiment was conducted to evaluate the effect of a mild feed restriction during this period with and without an ACTH blocking agent (metyrapone) on stress responses [16].

White Plymouth Rock chickens placed under $60 \%$ feed restriction or ad libitum feeding, with or without metyrapone treatment, from 4 to 6 days of age were subjected to either 8 or 24 hours feed deprivation at 36 days of age. Chicks subjected to the neonatal $60 \%$ feed restriction (60R) but not those provided metyrapone during the procedure $(60 \mathrm{M})$ had elevated heterophil/lymphocyte $(\mathrm{H} / \mathrm{L})$ ratios. However, there was no difference in plasma corticosterone and $\mathrm{ACTH}$ responses between $60 \mathrm{R}$ and $60 \mathrm{M}$ chicks. Except for increases in $\mathrm{H} / \mathrm{L}$ and plasma corticosterone concentrations among ad libitum fed $(\mathrm{AL})$ and $60 \mathrm{M}$ chickens, there was no indication of a stress response attributable to the subsequent 8 -hour fast. Feed withdrawal for 24 hours did not cause rises in $\mathrm{H} / \mathrm{L}$ ratios or plasma levels of corticosterone of chicks that had been subjected to early $60 \%$ feed restriction with non-metyrapone treated feed. In contrast, chicks of other regimens had elevated $H / L$ and plasma corticosterone responses when exposed to similar 
procedures. Except for those fed ad libitum during the neonatal stage, circulating levels of $\mathrm{ACTH}$ declined following the 24-hour fast. These data suggest that a critical period may exist during this early posthatch period that facilitates adaptation to stressors later in life.

\section{Feed Particle Size and Performance}

The experiments discussed in this section were orchestrated with those discussed in the previous sections. They were designed to examine practical ways to address via nongenetic means the supply-demand interface. One experiment [9] attempted to quantify effects of "degree" of pelleting on performance. In the first trial, diets were prepared by mixing together mash composed mainly of corn with soft pellets or soft pellets mixed with hard pellets. The second trial was similar except that the mash was composed mainly of sorghum rather than corn.

Pelleting degrees (PD) were as follows: $(0)$ mash, $(0.5)$ mixture of soft pellets and mash 1 to 1 , (1) soft pellets pelleted once, (1.5) mixture of soft and hard pellets 1 to 1 , (2) hard pellets pelleted twice. Eating, drinking, standing, and sitting behaviors were recorded in both trials, while in the second trial, data were also obtained for digestive enzymes and weights and lengths of digestive organs. Feed intake and body weight gain were related to the degree of pelleting in a curvilinear manner. PD had a positive effect up to a peak (1 to 1.5 PD), after which its effect decreased. Feed efficiency was not related to PD. In the first trial feed efficiency of PDs 1 to 2 were superior to PDs 0 to 0.5 and in the second trial PDs 1.5 to 2 were superior to PD 0 . Pelleting reduced relative gizzard weight and increased the relative abdominal fat weight. Content of the 
crop was not affected by PD, whereas that of the proventriculus was lowest in the PD group. Gizzard content was inversely related to PD. Pelleting reduced the length of the jejunum and ileum, which were shortened by about $15 \%$ with PDs 1 to 2, compared to PD 0 . The weight/length ratio of the jejunum and ileum tended to increase with increasing PD to a peak at PD $1 \cdot 5$, and to decrease thereafter. Pelleting reduced trypsin activity in the pancreas and amylase activity in the intestinal content. Also, chicks fed pelleted diets sat more and spent less time eating than those fed mash.

Effects of diets prepared with corn ground in a hammer mill and sieved to obtain coarse, medium, and fine particle size fractions, on broiler performance from hatch to 7 days and from 7 to 21 days of age were reported [10]. Five diets differing in geometric mean diameter (CMD) or geometric standard deviation (CSD) were assayed. The best performance in both age groups was obtained with the diet prepared from medium corn. In this diet the CMD was $0.769 \mathrm{~mm}$, quite similar to those of two other diets (GMD 0.793 and 0.706 obtained by mixing the original corn with 18 or $36 \%$ "fine" fraction) with which lower performance was obtained. The uniformity of the particle size (described by the GSD) also had a positive effect on performance. The improved performance obtained with the diet containing the medium corn particle size could be partly attributed to lower GSD, which was 1.63 vs about 2.00 in the other diets.

Effects of diets prepared with corn, wheat, or sorghum ground in a hammer mill and sieved to obtain coarse, medium, and fine particle size fractions on broiler performance from hatch to 7 days of age and from 7 to 21 days of age were studied [11]. The effect of particle size on the size and $\mathrm{pH}$ of the gastrointestinal organs was also 
determined. The three textures obtained for each grain were uniform in geometric mean diameter (GMD) and geometric standard deviation (GSD). Grain by texture interactions were not significant. Independent of grain source, the best performance was obtained with diets prepared from the medium texture. In these diets, the GMD of the grains varied from 1.13 to $1.23 \mathrm{~mm}$ and the GSD from 1.19 to 1.35 . The fine fraction (GMD 0.57 to $0.67 \mathrm{~mm}$ ) resulted in the poorest performance and that resulting from the coarse fraction (GMD 2.01 to $2.10 \mathrm{~mm}$ ) was intermediate. At 7 and 21 days of age, gizzard weight and content were positively related to particle size. At 21 days of age, duodenum weight and content was highest in chicks fed the fine diets. The $\mathrm{pH}$ of gizzard contents decreased with increasing grain particle size, whereas it increased for that of the small intestinal contents. Grain effects were also observed on the GIT where gizzard weight was greatest in the corn-fed birds and wheat-fed chicks had heavier intestines and intestinal contents. After overnight feed deprivation, feed consumption by 7 day-old chicks was related to the particle size during the first 2 hours of refeeding, with greater intake of medium and coarse grains.

Additional experiments [12] were conducted to study the effect of particle size obtained by grinding wheat and sorghum in a hammer mill $(H M)$ or roller (RM) on broiler performance. Broilers were fed a mash diet or crumbles to 28 days of age then pellets to 49 days. When fed as a mash, diets produced with RM-ground grain improved performance. The effect of grinding was additive to that of pelleting. The interactive effects observed between grinding method and the form of the feed on body weight and feed intake were explained by the higher response to pelleting when grains were ground 
in a $H M$ vs a RM. The positive effect of grinding in a RM on feed utilization was additive to that of pelleting. Although responses of females to feed texture was less pronounced than that of males, feeding pellets to males increased mortality due to ascites threefold, whereas grinding method had no effect. Females were less susceptible to ascites than males, and feed texture had no effect in this respect. The effects of grinding and pelleting on segments of the GIT were additive. The main effects of feed texture were increases in weight and that of its contents following HM-grinding and a decrease in following pelleting, with no interactions between the two.

This series of experiments provided insights for ways to circumvent the limitations imposed on chicks from the maturation of the digestive system. This, in turn, will enhance broiler performance.

\section{PUBLICATIONS}

1. Nitsan, Z., E. A. Dunnington, and P. B. Siegel, 1991. Organ growth and digestive enzyme levels to 15 days of age in chickens differing in body weight. Poultry Sci. 70:2040-2048.

2. O'Sullivan, N. P., E. A. Dunnington, and P. B. Siegel, 1991. Relationships among age of dam, egg components, embryo lipid transfer, and hatchability of broiler breeder eggs. Poultry Sci. 70:2180-2185.

3. O'Sullivan, N. P., E. A. Dunnington, and P. B. Siegel, 1992. Correlated responses in lines of chickens divergently selected for fifty-six-day body weight. 1. Growth, feed intake, and feed utilization. Poultry Sci. 71:590-597.

4. O'Sullivan, N. P., E. A. Dunnington, A. S. Larsen, and P. B. Siegel, 1992. Correlated responses in lines of chickens divergently selected for fiftysix-day body weight. 2. Organ growth, deoxyribonucleic acid, ribonucleic acid, and protein content. Poultry Sci. 71:598-609. 
5. O'Sullivan, N. P., E. A. Dunnington, A. S. Larsen, and P. B. Siegel, 1992. Correlated responses in lines of chickens divergently selected for fiftysix-day weight. 3. Digestive enzymes. Poultry Sci. 71:610-617.

6. Nir, I., Z. Nitsan, and M. Mahagna, 1993. Comparative growth and development of the digestive organs and of some enzymes in broiler and egg type chicks after hatching. Brit. Poult. Sci. 34:523-532.

7. Noble, D. O., E. A. Dunnington, and P. B. Siegel, 1993. Ingestive behavior and growth when chicks from lines differing in feed consumption are reared separately or intermingled. Applied Anim. Behav. Sci. 35:359368.

8. Mahagna, M., I. Nir, and Z. Nitsan, 1994. Influence of the presence of 3day-old chickens on the behaviour of meat and egg-type posthatch counterparts. Appl. Anim. Behav. Sci. 40:143-152.

9. Nir, I., Y. Twina, E. Grossman, and Z. Nitsan, 1994. Quantitative effects of pelleting on performance, gastrointestinal tract and behaviour of meattype chickens. Brit. Poult. Sci. 35(in press).

10. Nir, I., G. Shefet, and Y. Aaroni, 1994. Effect of particle size on performance. 1. Corn. Poultry Sci. 73:45-49.

11. Nir, I., R. Hillel, G. Shefet, and Z. Nitsan, 1994. Effect of particle size on performance. 2. Grain texture interactions. Poultry Sci. 73:781-791.

12. Nir, I., R. Hillel, I. Pitichi, and G. Shefet, 1994. Effect of particle size on performance. 3. Grinding pelleting interactions. Poultry Sci. (in press).

13. Mahagna, M., I. Nir, M. Larbier, and Z. Nitsan, 1995. Effect of age and exogenous amylase and protease on development of the digestive tract, digestive enzyme activity and digestibility of nutrients in young meattype chicks. French Annals (submitted).

14. Turro, I., Z. Nitsan, M. Picard, E. A. Dunnington, and P. B. Siegel, 1995. Removal of residual yolk at hatching influences food choice and feeding activity in lines of chickens selected for high or low juvenile body weight. Reprod., Nutrition \& Develop. (in press).

15. Nitsan, Z., I. Turro, G. Liu, E. A. Dunnington, and P. B. Siegel, 1995. Intubation of weight-selected chicks with soy-oil or residual yolk: Effect on early growth and development. Poultry Sci. (submitted). 
16. Zulkifli, I., H. S. Siegel, M. M. Mashaly, E. A. Dunnington, and P. B. Siegel, 1995. Inhibition of adrenal steroidogenesis, neonatal feed restriction and pituitary-adrenal axis response to subsequent fasting. Gen. Comp. Endocrin. (submitted).

\section{Evaluation of Research Achievements}

Research conducted during the course of this project addressed all objectives stated in the original proposal. Effective transfer of techniques and preliminary results among the involved laboratories during the first year provided direction for the second year which, in turn, laid the foundation for experiments conducted during the final years. The original view was that with broilers reaching market weights at younger ages, the first two weeks posthatch were becoming increasingly important. This was the period when chicks underwent transition from a primarily lipid-based metabolism at hatch to one that was primarily carbohydrate-based from external food sources. Results showed that not only were times just prior to hatch and the first week posthatch critical to subsequent growth and development, but that genetic and husbandry manipulations during this period had positive effects on the efficiency of broiler performance. An awareness of what may and may not be feasible, and realization of a need to optimize relationships between supply and demand organs has facilitated greater focus on the first week posthatch which is an increasingly larger segment of the life of a broiler. Positive responses from those in the poultry industry to our publications reflect their desire to apply findings that resulted from this project. 